\title{
Design of Efficient Flyback Switching Power Supply Based on LM5021
}

\author{
HOU Xingang ${ }^{1, a}$, WANG Lei ${ }^{1, b}$, CHEN Heng ${ }^{1, c}$, and CHEN Jinglei $i^{1, d}$ \\ ${ }^{1}$ Xijing University, Xi'an Shaanxi, China ,710123

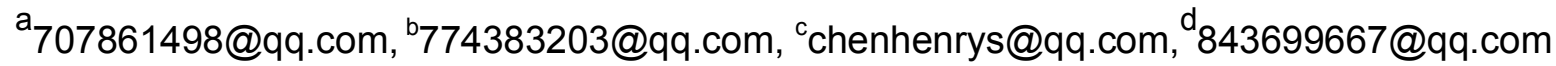

Keywords: flyback switching power supply; LM5021; current-limiting circuit; transformer Abstract. The paper introduces the process of designing an efficient flyback switching power supply on basis of LM5021 chip. It describes the process as the following 3 steps:1) designing a current-limiting circuit; 2) calculating the parameters of the transformer for the switching power supply and designing a feedback circuit as well as putting forward a new idea in accordance with the efficient self-driving circuit; 3 ) conducting prototyping test to prove the validity and realizability of the design.

\section{Introduction:}

Nowadays most studies of the switching power supply focus on the isolated power supply, such as a kind of ordinary flyback switching power supply studied in the articles [1] and a design of control circuits for the flyback switching power supply studied in the articles[2], both of which are based on the switching driver chip of UC3842. However, the paper discusses a design of simpler switching power supply with more useful and stable features in line with a TI company's current-type control chip LM5021[3], which is more efficient in utilizing energy, can apply soft startup and has a more matured market environment compared to the former.

The paper presents the designing process from 3 points:1)Learning the working principle of LM5021;2) Designing a transformer, which is the core device of the switching power supply, and giving forward an efficient method based on a self-drive circuit;3) And verifying the feasibility and practicability of the circuit designed by experiment.

\section{Basic parameters in designing an efficient flyback switching power supply}

The working principle [4] of the flyback converter is shown as Fig.1, where the input voltage range is $18 \sim 36 \mathrm{~V}$ and the nominal voltage is $24 \mathrm{~V}$. When the MOS is turned on, the output voltage is $15 \mathrm{~V}$, the output power is $30 \mathrm{~W}$, the output current is $2 \mathrm{~A}$, the operating temperature is $-45^{\circ} \mathrm{C} \sim 85^{\circ} \mathrm{C}$, the circuit load stability is less than $0.02 \%$, and the output ripple is less than $10 \mathrm{mV}$.

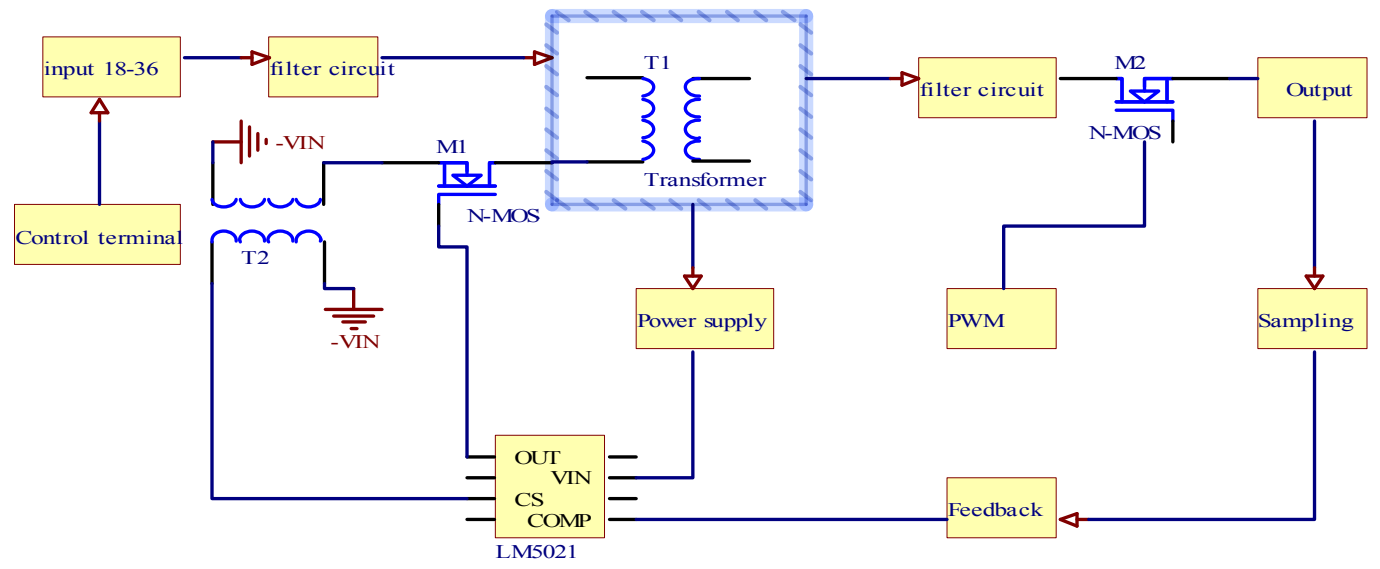

Fig.1 Overall block diagram of the power supply design

One of LM5021's biggest advantages is to realize the soft-start of the power supply so that the power conversion circuits can slowly reach the steady-state operating point, reducing the impact of 
starting and surge current. The LM5021 chip is able to provide a maximum current of $25 \mu \mathrm{A}$ to charge C15 at SS(as shown in Figure 2). The voltage ramp of C15 rises to limit the rate of rise for COMP, hence limiting rising in amplitude of the output pulse duty ratio to finish soft startup. Once the power supply starts, LM5021 can work through a lower starting current, ensuring high efficiency in using the power source.

Tab.1 The pin function of LM5021

\begin{tabular}{|l|l|}
\hline Name & \multicolumn{1}{|c|}{ Function } \\
\hline COMP & output of the feedback circuit isolated by optocouplers to control LM5021 \\
\hline VIN & input of an internal bias circuit \\
\hline VCC & Output of an internal bias circuit \\
\hline OUT & PWM output \\
\hline GND & ground return \\
\hline CS & current sense input \\
\hline RT & oscillator timing resistor pin and synchronization input \\
\hline SS & soft-start/hiccup time \\
\hline
\end{tabular}

\section{Current-limiting circuits}

The pin of CS chip used as the current sensor in the paper with a typical output voltage of $0.5 \mathrm{~V}$, when the input voltage is above $0.5 \mathrm{~V}$, the OUT pin controlled by CS chip stops output, and the over-current protection is triggered.

As shown in Figure 2, the current is sampled when MOS is turned on to allow the current to go through the transformer.and the higher harmonic is filtered when the current goes through the RC low-pass filter network. Then the CS chin gets a steady signal, protecting the circuit. In order to avoid a sudden rise of the voltage caused by leakage inductance when the switch is turned off, a buffer formed by R1, C1 and D4 is designed to make the power supply can work in the normal working range.

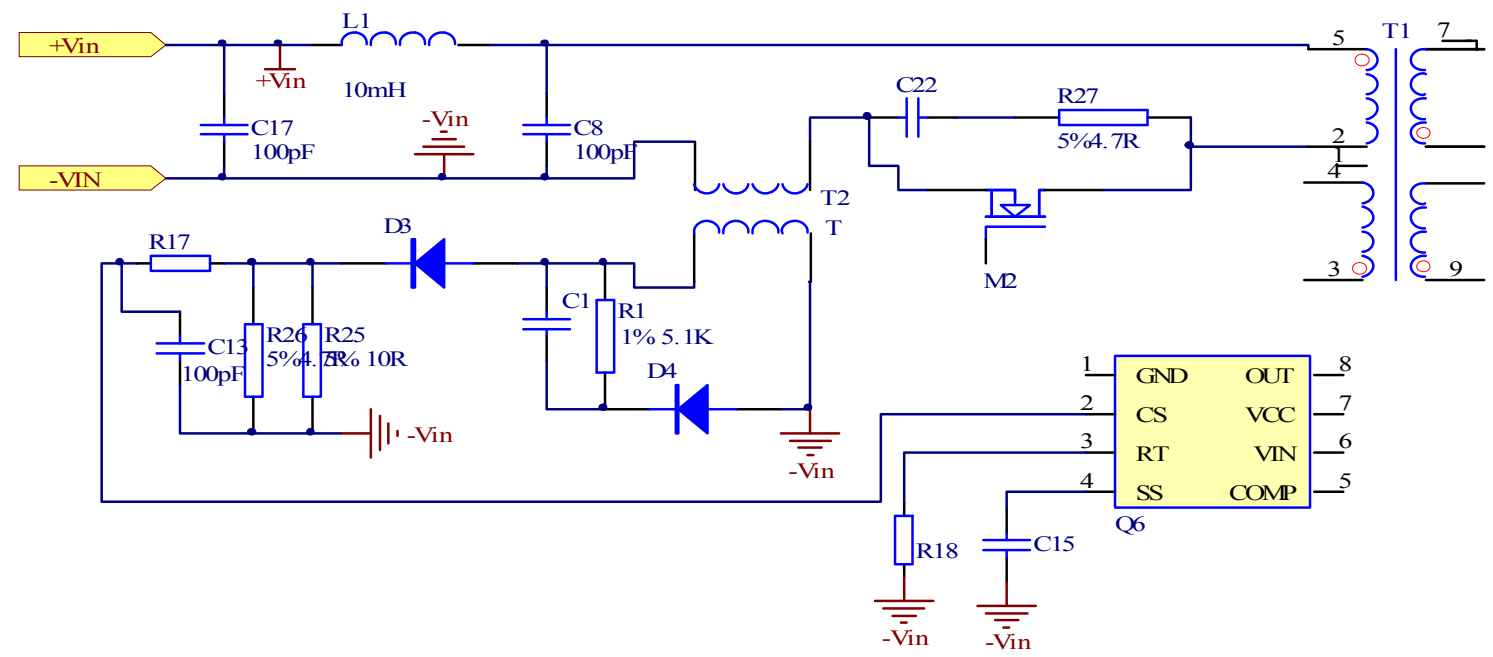

Fig.2 Current limiting circuit

\section{The design of the transformer}

As the core of the switching power supply, the flyback transformer is actually a coupled inductor. 
Because it can stock and transmit power and transform voltage, its design in the circuit is also very important[5]. The details for designing the transformer is as below.

\section{The primary inductance of the power switching transformer:}

The voltage ratio is obtained as:

$$
k=\frac{U_{s \max }-U_{i n \max }}{U_{o}}=\frac{60-36}{15}=1.6
$$

Where $U_{s \max }$ is the minimum working voltage, $U_{i n \max }$ is the maximum input voltage and $U_{o}$ is the output voltage.

At this point the primary side peak current of the transformer is obtained as:

$$
I_{p}=\frac{2 I_{o}}{k\left(1-D_{\max }\right)}=4.54(\mathrm{~A})
$$

Then the inductance for the primary side of the transformer is calculated as:

$$
L_{1}=\frac{D_{\max } U_{i n \min }}{I_{P} f_{s}}=\frac{D_{\text {max }}\left(1-D_{\max }\right) k U_{i n \min }}{2 I_{o} f_{s}}=6.8(\mu \mathrm{H})
$$

\section{The specifications of the magnetic core}

The transformer is designed in accordance with the $A_{p}$ method and its primary and secondary coils can be regarded as two inductors. The calculation as below:

$$
A P_{p}=\frac{6.33 \times L_{1} \times I_{P} \times d_{w}^{2} \times 10^{8}}{\Delta B}
$$

According to the skin effect, the diameter of the edge winding wire is $d_{w}=0.5 \mathrm{~mm}$, the variation of the magnetic induction is $\Delta B=\frac{B_{\max }+0}{2}, B_{\max }=0.2 \mathrm{~T},\left(B_{\max }\right.$ is less than 0.3$)$.

$$
A P=4 A P_{p}=0.196\left(\mathrm{~cm}^{4}\right)
$$

The model of the magnetic core is determined by the size and volume of the power supply. A longer EP20 of TDK is chosen as an optional magnetic core to allow enough length for winding the frame[6]. According to the data provided by TDK, the calculation is:

$$
A_{e} A_{w}=78 \times 55.4=4321\left(\mathrm{~mm}^{4}\right)=0.4321\left(\mathrm{~cm}^{4}\right)
$$

Because $0.196<0.4321$, the magnetic coil is suitable to the frame.

\section{The air gap length of the iron core}

For the same DC bias, reducing the density of working flux and increasing the saturation resistance can be achieved by widening the air gap of the flux in the iron core. The specific width of air gap is calculated as:

$$
L_{g}=\frac{0.4 \pi L_{1} I_{p} \times 10^{8}}{A_{e} B_{\max }^{2}}=0.55(\mathrm{~mm})
$$




\section{The winding turns of the transformer}

The primary winding turns of the transformer $N_{1}$ is 9 . The calculation is as below:

$$
N_{1}=\frac{L_{1} \times I_{p}}{A_{e} B_{\max }}=8.77(\mathrm{~T})
$$

The secondary winding turns of the transformer $N_{2}$ is also 9 . The calculation is as follows:

$$
N_{2}=\frac{N_{1}\left(U_{o}+V_{d}\right)\left(1-D_{\max }\right)}{U_{i \min } D_{\max }}=9.16(\mathrm{~T})
$$

The feedback winding turns $N_{3}$ is 6 . The calculation is:

$$
N_{3}=\frac{N_{1} \times V_{i n}}{U_{\text {in }}}=6(\mathrm{~T})
$$

When $N_{3}$ is $6, V_{C C}$ is the power supply for LM5021, the rang for $V_{\text {in }}$ is $8.5 \mathrm{~V} \sim 30 \mathrm{~V}$ based on the document, and the standard $V_{i n}$ is $12 \mathrm{~V}$. Therefor, the auxiliary winding turns $N_{4}$ is 7 by calculation.

$$
\frac{N_{2}}{N_{4}}=\frac{U_{o}}{V_{4}} \text { namely } N_{4}=\frac{N_{2} \times V_{4}}{U_{o}}=7.2(\mathrm{~T})
$$

In order to reduce the leakage inductance, the transformer in this paper winds in means of sandwich type. As shown in Figure 3, the black coil $N_{1} 1 / 2$ stands for the primary winding of the transformer, which is in the first and the fourth layers with 9T for each; the white coil $N_{2} 1 / 2$ represents the secondary winding $N_{2}$ in the second and the fifth layers with 9T for each; the third layer stands for the auxiliary winding $N_{4}$ with 6T; and the sixth layer represents the feedback winding $N_{3}$ with 7T. Thus the the first and the fourth layers, and the second and the fifth layers can be seen as two compact capacitors for absorbing the noise of the transformer. And the double coils spread the current and increase the power, preventing the transformer from burning. What's more, the $3 \mathrm{M}$ barrier tape between the coils protects devices from scratching.

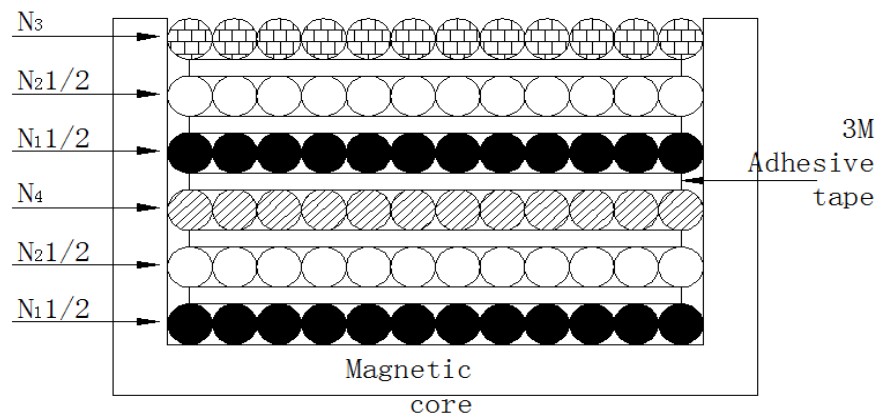

Fig.3 Transformer winding diagram

\section{The designs for the self-drive circuit and the voltage feedback loop}

\section{The self-drive circuit.}

The flyback circuit with a general switching power supply would connect a break over diode at the output end M1 shown as Figure 4, but this kind of circuit add the load to the diode to drop voltage with the output efficiency of $85 \%$. In order to increase the efficiency, a self-drive circuit can be connected to the output end as Figure 4 to lower the consumption of the output end. The current from the auxiliary end $N_{4}$ flows through D6 to charge SP6018, which generates PWM supplied to the grid 
of MOS, when MOS is turned on, the output voltage is generated, hence reducing the loss caused by the voltage drop difference of the diode.

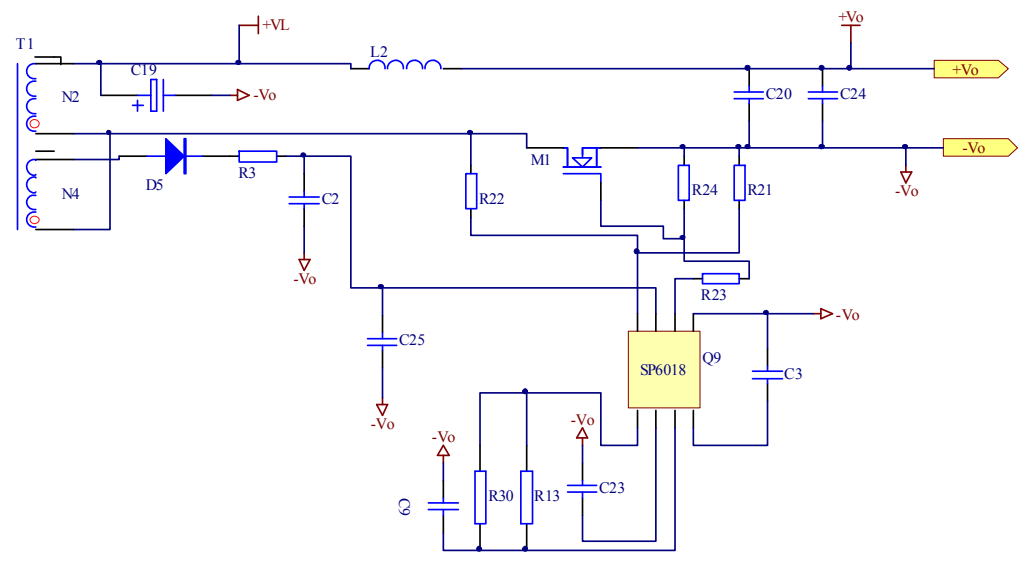

Fig.4 The schematic of self driving circuit

\section{The voltage feedback loop}

Due to a higher requirement for the voltage regulation and load regulation, the high-precision reference TL432 and optocoupler TLP291 are used in the design, which form an external error amplifier to isolate the output voltage and primary coil(R9 and $\mathrm{C} 10$ as shown in Figure 5 are necessarily used as compensation frequency for TL432). In the feedback circuit equipped with TL432, TL431 and the voltage regulator in parallel connection replace the voltage-regulator tube to regulate the output voltage. Although the circuit is complicated, the voltage regulation performance is best. When the output voltage rise above the defined output amplitude, the optocoupler conduction rate increases, the COMP pin of LM5021 is taken down, MOS is turned off and stops output. When the output voltage recovers to the defined amplitude, the chip works properly and the overload protection is triggered. When the output voltage is lower than the amplitude, the error amplifier's output voltage drops so that the chip adjusts PWM and changes the duty ratio, and the output voltage begins to stabilize. The information above is shown in Figure 5.

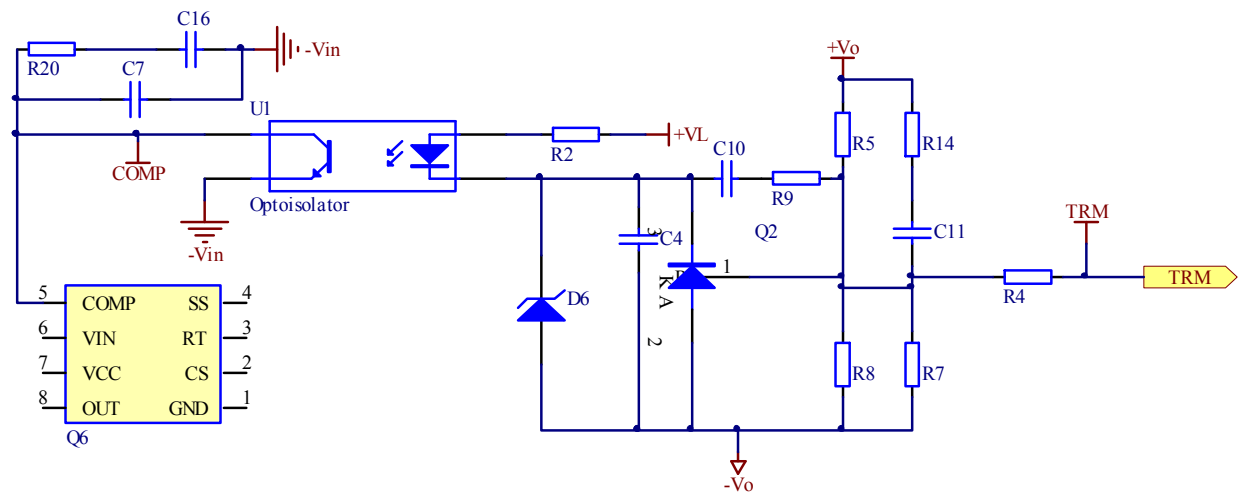

Fig.5 Feedback circuit schematics

According to the technical data of TL432, the reference voltage is $2.5 \mathrm{~V}$, the reference input current is $2 \mu \mathrm{A}$, and the current of $R 9$ is usually 100 times of the former. That is:

$$
R 9 \leq \frac{2.5}{0.002 \times 100}=12.5(\mathrm{k} \Omega)
$$

$R 9$ is $10 \mathrm{~K}$ based on the actual conditions and needs. The high-precision reference resistance is given as : 


$$
R 7 / / R 8=\left(U_{o}-V_{r e f}\right) \frac{R 9}{V_{r e f}}=50(\mathrm{k} \Omega)
$$

According to the technical data of TLP291, the breakover current $I_{F}$ is $50 \mathrm{~mA}$, the breakover voltage $V_{F}$ is $1.2 \mathrm{~V}$ and $\mathrm{D} 6$ is a voltage-regulator diode. Then the calculation is:

$$
R 2=\frac{V_{L}-V_{r e f}-V_{F}}{I_{F}}=\frac{U_{o}-V_{r e f}-V_{F}}{I_{F}}=226(\Omega)
$$

\section{The results of the experiment}

As shown in Figure 6, according to brassboard designed in the design, the following data is obtained. When the output end is a diode, the nominal input voltage is $24 \mathrm{~V}$, the output power is $30 \mathrm{~W}$, then the input current is $1.46 \mathrm{~A}$, and the power efficiency is up to $85.6 \%$; when the output end is a MOS tube, the input voltage is $24 \mathrm{~V}$, the output power is $30 \mathrm{~W}$, then the input current is $1.36 \mathrm{~A}$, and the power efficiency is up to $91.9 \%$. The result tells that the application of a self-drive increases $5 \%$ for the efficiency. Figure 7 is the oscillogram for the driver tube of MOS, from which the experiment effect is good and practical.

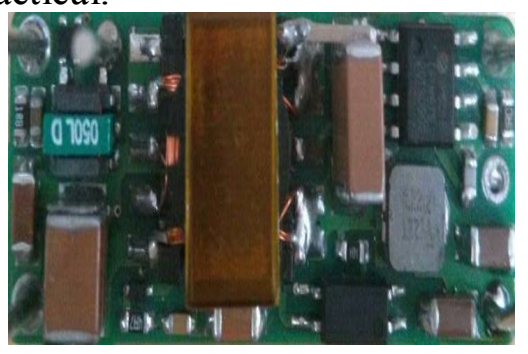

Fig.6 Breadboards

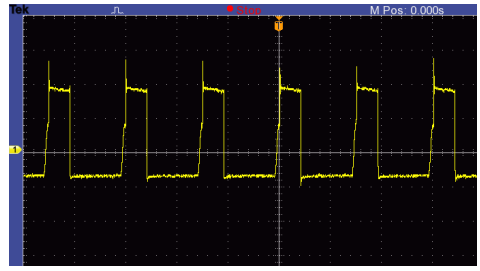

Fig.7 Waveform figure

\section{Conclusion}

The paper introduces the design of an efficient DC-DC switching power supply, and describes the process as follows: the designs of a current-limiting circuit, transformer and feedback circuit, and a self-drive circuit increasing the efficiency and an experiment to test the feasibility of the circuit, providing more choices for life.

\section{References:}

[1]LIU Ning, WANG Youhuan, XIA Dongwei.Design of the Super Wide Input Voltage Range Flyback Power Supply[J].Journal of Power Supply,2014,12(5):92-96.

[2]WANG Xinxing,LI Hong.Asingle-chip flyback power based on UC3842 integrated controller[J]. Chinese Journal of Power Sources,2013,37(8):1442-1445.

[3]Texas Instruments.LM5021.PDF[Z].USA:Texas Instruments.2013.

[4]GUAN Xiaolei,LIU FUli,CHI Shuang,WEI Jian.The Control-Loop Design Example of Flyback SMPS Based on UC3844 [J].Telecom Power Technologies,2010,27(5):53-58.

[5]ZHANG Housheng.Novel Design Method of Flyback Transformer and Its Snubber Circuit[J].

.Electric Drive,2010,40(11):49-52.

[6]ZHANG Zhongshi, WANG Wei, CHEN Wen. Calculation of Air-gap Quantity of Switching Power Supply Transformer Core[J]. Magnetic Materials And Devices, 2008, 39(1):53-68. 